\title{
Tumor fibroso solitario gigante de pleura. Serie de casos
}

\section{Giant Solitary Fibrous Tumor of the Pleura. Case Series}

\author{
${ }^{1}$ Facultad de Medicina y Ciencias de la Salud, Universidad del Rosario, \\ Bogotá, Colombia \\ 2 Departamento de Patología, Hospital Universitario Mayor Mederi \\ Universidad del Rosario, Bogotá, Colombia \\ ${ }^{3}$ Departamento de Cirugía de Tórax, Hospital Universitario Mayor \\ Mederi Universidad del Rosario, Bogotá, Colombia \\ ${ }^{4}$ Departamento de Radiología, Hospital Universitario Mayor Mederi \\ Universidad del Rosario, Bogotá, Colombia
}

Laura Marcela Fino-Velásquez ${ }^{1}$ Paulina Ojeda-León ${ }^{2}$ Julio César Granada-Camacho ${ }^{3}$

Mario López-Ordoñez ${ }^{3}$ Mónica Parrado-Delgado ${ }^{3}$ jorge Alberto Carrilo-Bayona ${ }^{4}$

\author{
Address for correspondence Jorge Alberto Carrillo Bayona, MD, \\ Departamento de Radiología, Hospital Universitario Mayor, Calle 24 \\ \#29-45, Bogotá, Colombia (e-mail: jorcarbay@hotmail.com).
}

Rev Argent Radiol 2021;85:98-105.

\section{Resumen}

\section{Palabras Clave \\ - tumor fibroso solitario \\ - pleura \\ - neoplasia pleural}

\section{Abstract}

received

December 11, 2017

accepted

July 20, 2020

published online

March 29, 2021
Introducción El tumor fibroso solitario de pleura (TFSP) es una neoplasia poco frecuente, con aproximadamente 1.000 casos reportados en la literatura mundial. La aproximación diagnóstica inicial se realiza con estudios imagenológicos.

Métodos De forma retrospectiva, se recopilaron cuatro casos de pacientes con TFSP gigante operados en nuestra institución. Se describen las características sociodemográficas, clínicas, imagenológicas, macroscópicas y microscópicas de cada caso. Resultados Todos los pacientes de la serie cursaron con manifestaciones clínicas, con un promedio de 23,75 meses de evolución. El $50 \%$ de los tumores se localizaron en la cavidad pleural derecha y el $50 \%$ en la izquierda. En tomografía computarizada (TC) de tórax, los cuatro casos se presentaron como una masa sólida, de densidad heterogénea, con diámetros mayores entre 17 y $22 \mathrm{~cm}$ y contornos variables (lisos en tres casos y lobulados en un paciente). Se observaron calcificaciones intratumorales en dos casos y derrame pleural en tres pacientes. En cirugía, todas las masas presentaron pedículos. El análisis histológico e inmuno-químico confirmó la naturaleza benigna de tres casos y malignidad en una de las neoplasias.

Conclusiones Los TFSP generalmente son benignos y de buen pronóstico. Sin embargo, entre 10 y $20 \%$ de esos tumores son malignos. Las imágenes diagnósticas pueden sugerir el diagnóstico de TFSP, pero la confirmación de la naturaleza de la lesión debe realizarse con el análisis histopatológico de toda la pieza quirúrgica.

Introduction Solitary fibrous tumor of the pleura (SFTP) is a rare neoplasm, with $\sim 1.000$ cases reported in the worldwide literature. The initial diagnostic approach is performed by imaging studies.
DOI https://doi.org/ $10.1055 / \mathrm{s}-0040-1721344$. ISSN $1852-9992$.

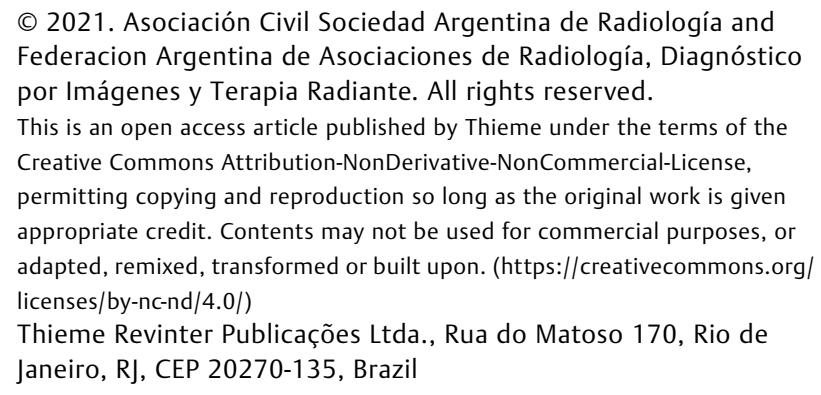

(C) 2021. Asociación Civil Sociedad Argentina de Radiología and Federacion Argentina de Asociaciones de Radiología, Diagnóstico por Imágenes y Terapia Radiante. All rights reserved.

This is an open access article published by Thieme under the terms of the Creative Commons Attribution-NonDerivative-NonCommercial-License, permitting copying and reproduction so long as the original work is given appropriate credit. Contents may not be used for commercial purposes, or adapted, remixed, transformed or built upon. (https://creativecommons.org/ licenses/by-nc-nd/4.0/)

Thieme Revinter Publicações Ltda., Rua do Matoso 170, Rio de Janeiro, RJ, CEP 20270-135, Brazil 


\section{Keywords}

- solitary fibrous tumor

- pleura

- pleural neoplasm
Methods Retrospectively, we collected four cases of patients with giant SFTP operated in our institution. The sociodemographic, clinical, imaging, macroscopic, and microscopic characteristics of each case are described.

Results All the patients in the series had clinical manifestations, with an average of 23.75 months of evolution. $50 \%$ of the tumors were located in the right pleural cavity and $50 \%$ in the left. In chest computed tomography (CT), the four cases presented as a solid mass, of heterogeneous density, with greater diameters between 17 and $22 \mathrm{~cm}$, and variable contours (smooth in three cases and lobulated in one patient). Intratumoral calcifications were observed in two cases and pleural effusion in three patients. In surgery, all masses presented pedicles. The histological and immunochemical analysis confirmed the benign nature of three cases and malignancy in one of them.

Conclusions SFTPs are usually benign and have a good prognosis. However, between 10 and $20 \%$ of these tumors are malignant. Diagnostic images may suggest the diagnosis of SFTP, but confirmation of the nature of the lesion should be made with the histopathological analysis of the entire surgical specimen.

\section{Introducción}

Los tumores fibrosos son neoplasias poco frecuentes, que pueden encontrarse en diferentes localizaciones que incluyen: vía aérea, tracto urinario, sistema nervioso, tejido óseo, órganos gastrointestinales y pleura. ${ }^{1}$

El tumor fibroso solitario de pleura (TFSP) es una neoplasia localizada, sin asociación a factores exposicionales. Por el contrario, el mesotelioma pleural es un tumor generalmente difuso, que con frecuencia se asocia a exposición a asbesto. ${ }^{2}$

La mayoría de TFSP son de comportamiento benigno. Sin embargo, el $10 \%$ de los TFSP son potencialmente malignos. Se considera que un diámetro mayor de $10 \mathrm{~cm}$ aumenta significativamente la probabilidad de malignidad. Los TFSP con diámetro mayor de $15 \mathrm{~cm}$ se describen en la literatura como gigantes. ${ }^{3,4}$

Presentamos cuatro casos de TFSP gigantes diagnosticados en nuestra institución ( - Tabla $\mathbf{1}$ ).

\section{Caso 1}

Mujer de 66 años que presentó disminución de la clase funcional, dolor torácico, tos con expectoración blanquecina y pérdida de peso de un año de evolución. Como antecedentes de importancia se anotan: exposición a biomasa asociada a enfermedad pulmonar obstructiva crónica e hipertensión pulmonar precapilar. La tomografía computarizada (TC) de tórax con contraste endovenoso demostró una masa extrapulmonar derecha (de origen pleural), con densidad heterogénea, la cual condicionaba atelectasia pasiva del parénquima pulmonar adyacente y era sugestiva de TFSP (-Fig. 1).

En cirugía, se confirmó la presencia de una masa pleural bipediculada (-Fig. 2), no encapsulada, de aspecto hemorrágico, con áreas necróticas. Microscópicamente, el tejido estaba constituido por fibras de colágeno y células fusiformes de núcleo oval con citoplasma no definido (-Fig.3).

\section{Caso 2}

Mujer de 71 años que se presentó con disnea, dolor torácico, ortopnea y edema de miembros inferiores de dos meses, así como tos con expectoración blanquecina de 10 días de evolución. Los antecedentes relevantes en la historia clínica incluyeron: EPOC con hipertensión pulmonar severa e insuficiencia cardíaca. La TC de tórax demostró una masa pleural sólida, de contornos lisos bien definidos ( - Fig. 1). Dada la sospecha imagenológica de TFSP, se resecó la lesión. El análisis histopatológico de la pieza quirúrgica confirmó la presencia de tumor fibroso de pleura, sin hallazgos morfológicos de malignidad.

\section{Caso 3}

Hombre de 50 años que cursó con cuadro de nueve meses de disnea, tos seca, pérdida de peso no cuantificada, mialgias y poliartralgias. La TC de tórax (—Fig. 1) demostró la presencia de una masa sólida, extrapulmonar, de densidad heterogénea, ricamente vascularizada, la cual era compatible con TFSP. Adicionalmente, se asociaba a atelectasia pasiva secundaria $\mathrm{y}$ a derrame pleural.

En cirugía, se confirmó la presencia de una masa pleural, irrigada por vasos sanguíneos de la pleura visceral (-Fig. 2). El análisis microscópico evidenció tumor constituido por fibras de colágeno y células fusiformes de núcleo oval y citoplasma no definido, con marcado pleomorfismo celular, núcleos gigantes, numerosas mitosis y focos de necrosis, que correspondía a tumor fibroso maligno de pleura (-Fig. 3).

\section{Caso 4}

Hombre de 80 años que consultó por dolor torácico episódico y disnea ocasional de seis años de evolución, con antecedentes de artritis reumatoide, hipertensión arterial e hipertensión pulmonar. 
Tabla 1 Características sociodemográficas, clínicas, imagenológicas, microscópicas, macroscópicas y hallazgos intraoperatorios de los cuatro casos de TFSP gigantes

\begin{tabular}{|c|c|c|c|c|c|}
\hline & & Caso 1 & Caso 2 & Caso 3 & Caso 4 \\
\hline \multirow[t]{2}{*}{ Paciente } & Edad (años) & 66 & 71 & 50 & 80 \\
\hline & Sexo & Femenino & Femenino & Masculino & Masculino \\
\hline \multirow{2}{*}{$\begin{array}{l}\text { Manifestaciones } \\
\text { clínicas }\end{array}$} & Sintomatología & $\overline{\text { Sí }}$ & Sí & Sí & Sí \\
\hline & $\begin{array}{l}\text { Tiempo de } \\
\text { evolución (meses) }\end{array}$ & 12 & 2 & 9 & 72 \\
\hline \multirow{9}{*}{$\begin{array}{l}\text { Factores } \\
\text { imagenológicos } \\
\text { (CT) }\end{array}$} & Lateralidad & Derecha & Derecha & Izquierda & Izquierda \\
\hline & Localización & Basal & Basal & Basal & Basal \\
\hline & Densidad & Heterogénea & Heterogénea & Heterogénea & Heterogénea \\
\hline & Bordes & Lisos & Lisos & Lisos & Lobulados \\
\hline & Vascularización & Sí & Sí & Sí & Sí \\
\hline & Calcificaciones & No & No & Sí & Sí \\
\hline & Pedículo & No & No & $\begin{array}{l}\text { Sí (hacia el } \\
\text { diafragma) }\end{array}$ & $\begin{array}{l}\text { Sí (hacia el } \\
\text { pericardio) }\end{array}$ \\
\hline & Derrame pleural & No & Sí & Sí & Sí \\
\hline & $\begin{array}{l}\text { Diámetro del tronco } \\
\text { de la AP }(\mathrm{mm})\end{array}$ & 33 & 36 & 24 & 36 \\
\hline \multirow{8}{*}{$\begin{array}{l}\text { Biopsia } \\
\text { prequirúrgica }\end{array}$} & Mitosis & No & No & No & No \\
\hline & Pleomorfismo & No & No & No & No \\
\hline & Necrosis & No & No & No & No \\
\hline & $\begin{array}{l}\text { Índice de } \\
\text { proliferación }\left(K_{i}-67\right)\end{array}$ & $<1 \%$ & $1 \%$ & $<1 \%$ & - \\
\hline & $\mathrm{BCL} 2$ & Positivo & Positivo & Positivo & Positivo \\
\hline & CD34 & Positivo & Positivo & Positivo & Positivo \\
\hline & S100 & Negativo & Negativo & Negativo & Negativo \\
\hline & CD99 & - & Negativo & Negativo & Positivo \\
\hline \multirow[t]{2}{*}{$\begin{array}{l}\text { Procedimiento } \\
\text { quirúrgico }\end{array}$} & Abordaje quirúrgico & $\begin{array}{l}\text { Toracotomía } \\
\text { anterolateral }\end{array}$ & $\begin{array}{l}\text { Toracotomía } \\
\text { posterolateral }\end{array}$ & $\begin{array}{l}\text { Toracofreno- } \\
\text { laparotomía }\end{array}$ & $\begin{array}{l}\text { Toracotomía } \\
\text { posterolateral }\end{array}$ \\
\hline & Pedículos & Múltiples & Único & Múltiples & Múltiples \\
\hline \multirow{2}{*}{$\begin{array}{l}\text { Análisis } \\
\text { macroscópico }\end{array}$} & Dimensiones & $190 \times 145 \times 95$ & $170 \times 150 \times 105$ & $200 \times 160 \times 120$ & $220 \times 170 \times 50$ \\
\hline & Peso (gramos) & 1350 & 1486 & 2000 & 2184 \\
\hline \multirow{12}{*}{$\begin{array}{l}\text { Análisis } \\
\text { microscópico }\end{array}$} & Cápsula & No & Sí & Sí & Sí \\
\hline & Mitosis & No & No & Sí & No \\
\hline & Pleomorfismo celular & No & No & Sí & No \\
\hline & Necrosis & Sí & Sí & Sí & No \\
\hline & Hemorragia & Sí & Sí & No & Sí \\
\hline & $\begin{array}{l}\text { Índice de } \\
\text { proliferación }\left(K_{i}-67\right)\end{array}$ & $20 \%$ & $20 \%$ & $0-60 \%$ & $0-30 \%$ \\
\hline & $\mathrm{BCL} 2$ & Positivo & Positivo & Positivo & Positivo \\
\hline & CD34 & Positivo & Positivo & Positivo & Positivo \\
\hline & Vimentina & Positivo & Positivo & Positivo & Positivo \\
\hline & AML & Negativo & Negativo & Negativo & Negativo \\
\hline & S100 & Negativo & Negativo & Negativo & Negativo \\
\hline & CD99 & Negativo & Negativo & Negativo & Negativo \\
\hline
\end{tabular}


Caso 1
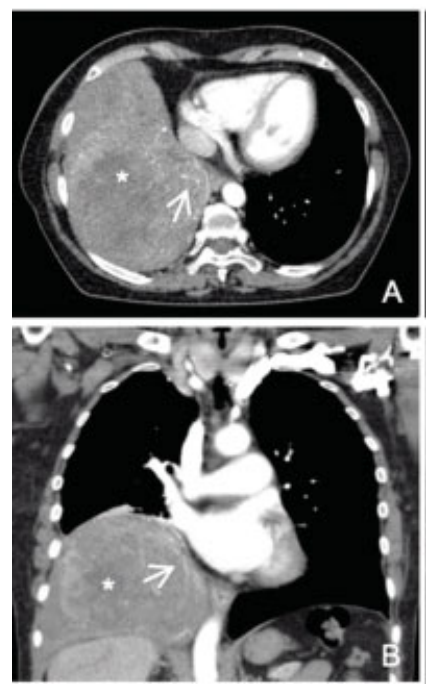

Caso 2
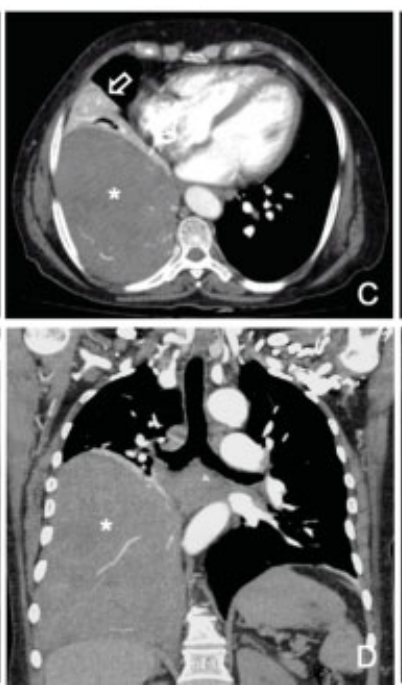

Caso 3
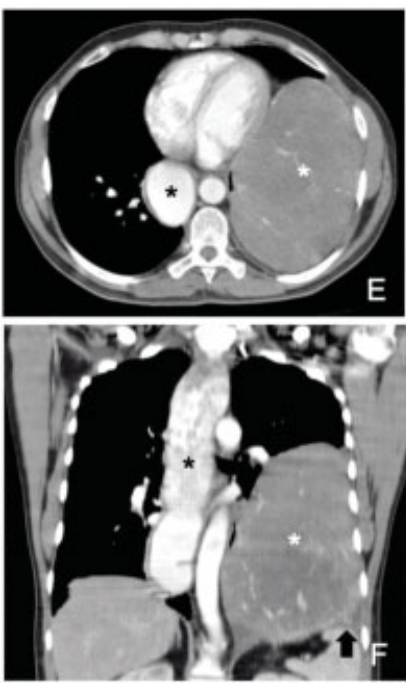

Caso 4
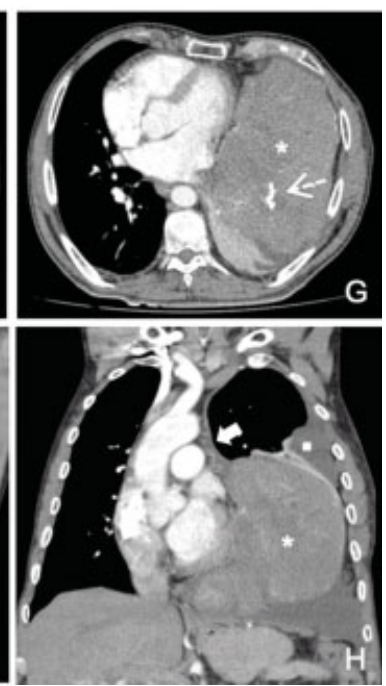

Fig. 1 TC de tórax con medio de contraste endovenoso, en ventana para mediastino. Caso 1: Reconstrucciones axial (A) y coronal (B), masa extrapulmonar, sólida, vascularizada y con densidad heterogénea (asterisco), la cual presenta múltiples estructuras vasculares en su interior (flechas blancas). Caso 2: Reconstrucciones axial (C) y coronal (D), tumor sólido vascularizado (asterisco), el cual es dependiente de la pleura y condiciona atelectasia del parénquima pulmonar adyacente (flecha hueca). Caso 3: Reconstrucciones axial (E) y coronal (F), masa hipervascularizada (asterisco blanco), de densidad heterogénea, la cual presenta escasas calcificaciones nodulares y pedículo vascularizado (flecha negra gruesa), como hallazgo incidental se identifica dilatación esofágica (asterisco negro). Caso 4: Reconstrucciones axial (G) y coronal (H), lesión sólida de contornos lobulados (asterisco) con pedículo vascular (flecha blanca gruesa) y calcificaciones intralesionales (flecha blanca discontinua), la cual rechaza las estructuras mediastínicas y se asocia a derrame pleural libre (cuadrado blanco).

La TC de tórax solicitada con sospecha de embolia pulmonar demostró masa sólida, extrapulmonar, basal izquierda, vascularizada, con calcificaciones, que desplazaba estructuras mediastínicas y se asociaba a atelectasia pasiva del parénquima pulmonar adyacente y líquido pleural libre (-Fig. 1). Hallazgos imagenológicos relacionados con TFSP.

En cirugía, se evidenció una masa dependiente de la pleura visceral con pedículos al pericardio y a la pleura visceral, asociado a derrame pleural libre (-Fig. 2). El análisis histopatológico demostró un tumor sólido, encapsulado, constituido por fibras de colágeno y células fusiformes de núcleo oval y citoplasma no definido.

\section{Discusión}

Las neoplasias originadas en la pleura pueden ser primarias o secundarias. Las lesiones primarias son poco frecuentes, representan menos del 5\% de los tumores de la pleura y se clasifican de acuerdo a su naturaleza (benignas o malignas) y a su origen (mesotelial, mesenquimal o linfoproliferativo) (-Gráfico 1). . $^{\text {, } 6}$

El TFSP es una neoplasia primaria que se origina en el $80 \%$ de los casos en la pleura visceral. Previamente se ha denominado mesotelioma localizado, fibroma submesotelial y hemangiopericitoma. ${ }^{1,7,8}$ Usualmente, se diagnostica en la sexta o séptima década de la vida y tiene una leve predilección por la población femenina. Su incidencia anual es de 2,8 casos por 100.000 habitantes. $^{2,7,9}$

El TFSP se origina de células fusiformes mesenquimales submesoteliales con diferenciación fibroblástica. ${ }^{2}$ Se considera que esa neoplasia se asocia a la fusión intracromosómica de NAB2 y STAT6, localizados en el cromosoma 12q. Generalmente, esos genes tienen sentidos opuestos, pero en los pacientes con TFSP tienen la misma orientación. ${ }^{10}$

El diagnóstico del TFSP puede ser incidental. Aproximadamente la mitad de los pacientes son asintomáticos. Las manifestaciones clínicas son inespecíficas e incluyen disnea, tos, dolor torácico, síntomas constitucionales y son más frecuentes en neoplasias de gran tamaño. ${ }^{7,11}$ Dado que en nuestra serie de casos se recopilaron casos de TFSP gigantes, la prevalencia de manifestaciones clínicas fue del $100 \%$. La sintomatología se instauró de forma subaguda-crónica y solo en un caso se presentaron síntomas sobreagregados de evolución aguda.

El 4\% de los pacientes con TFSP pueden cursar con hipoglucemia, esa asociación se ha denominado síndrome de Doege-Potter y está relacionada con la capacidad que tiene el tumor de producir factores de crecimiento similares a la insulina que condicionan hipoglucemia la cual desaparece cuando se reseca la lesión. Esa presentación es más frecuente en neoplasias de mayor tamaño o con alta tasa mitótica. ${ }^{12,13}$ Otras manifestaciones clínicas asociadas incluyen la acropaquia y la osteoartropatía pulmonar hipertrófica. $^{14}$

Las imágenes son una herramienta esencial en el diagnóstico de TFSP y pueden sugerir pronóstico, de acuerdo a características específicas como: tamaño, presencia de necrosis, derrame pleural asociado, entre otros. ${ }^{7,15}$

La radiografía de tórax es el primer estudio imagenológico que se realiza en pacientes con TFSP. El hallazgo marcador en la radiografía está constituido por 

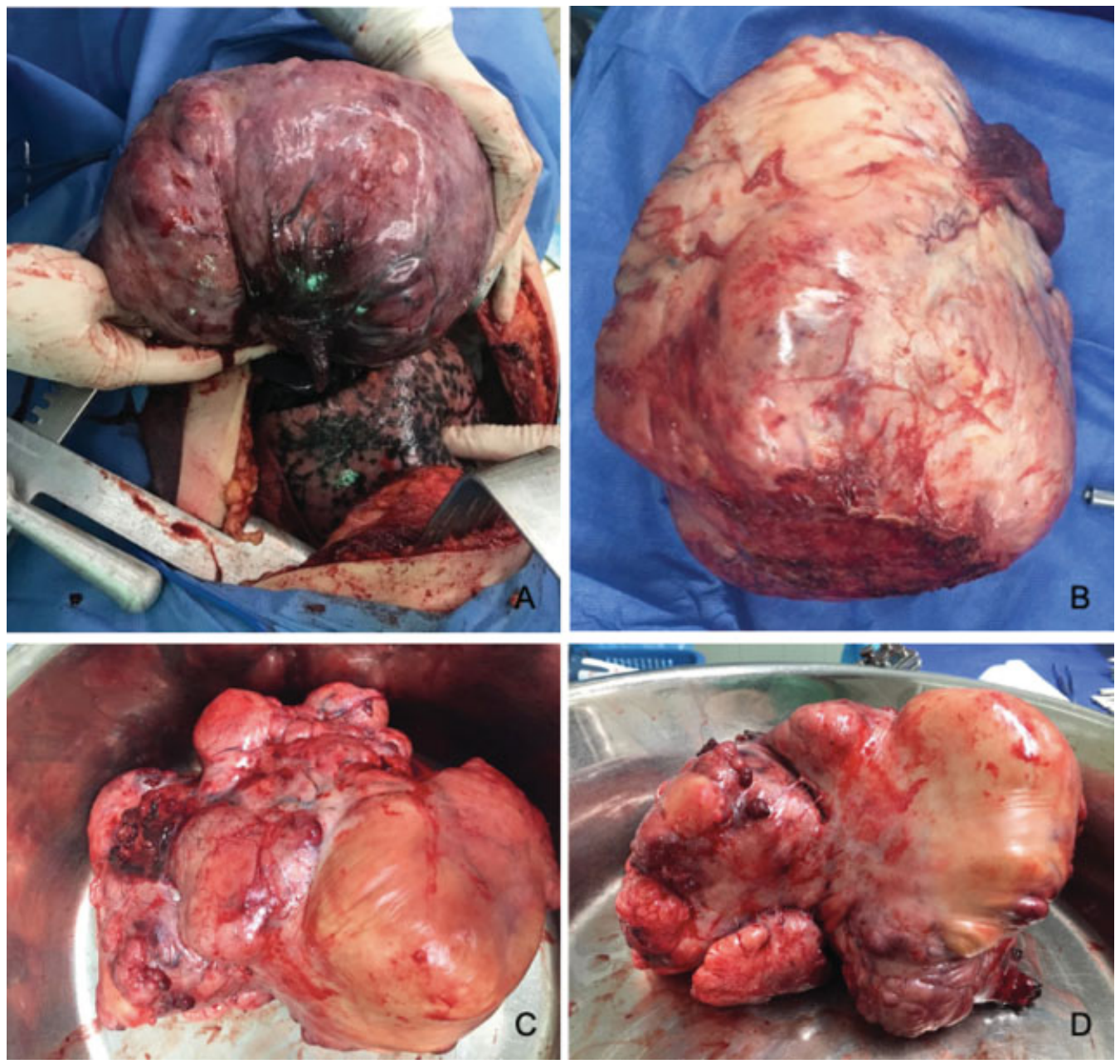

Fig. 2 Piezas quirúrgicas (TFSP). Caso 1(A): Masa bipediculada de aspecto hemorrágico con áreas necróticas, la cual era dependiente de la pleura. Caso 3 (B): Masa extrapulmonar de gran tamaño y contornos lisos, la cual estaba irrigada por vasos de la pleura visceral. Caso 4 (C y D): Masa pleural hipervascular, de aspecto nodular, con múltiples pedículos al pericardio y a la pleura visceral.
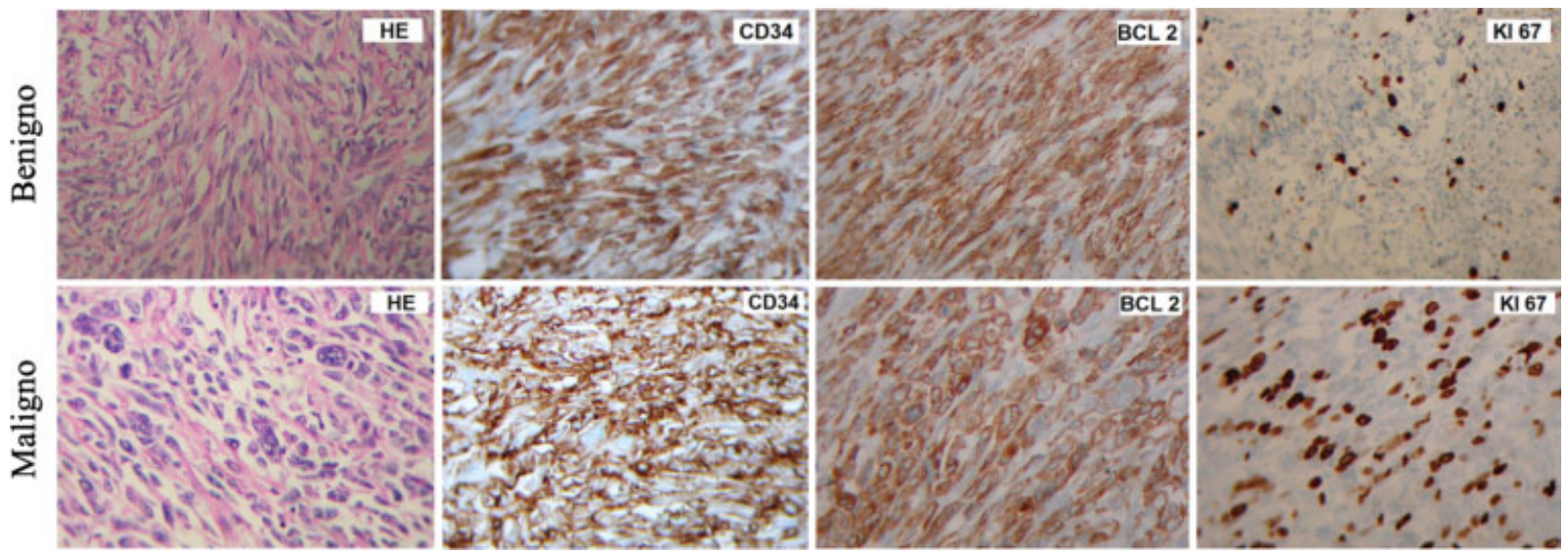

Fig. 3 Análisis inmunohistoquímico (40x) de TFSP benigno vs TFSP maligno. TFSP benigno (Caso 1): Coloración hematoxilina-eosina (HE), en la cual se evidencia tejido constituido por fibras de colágeno y células fusiformes de núcleo oval y citoplasma no definido sin evidencia de mitosis ni pleomorfismo celular, la inmunohistoquímica para CD34 y BCL 2 muestra inmunorreactividad de las células. El índice de proliferación medido con $\mathrm{K}_{\mathrm{i}}-67$ fue del 20\%. TFSP maligno (Caso 3): Coloración hematoxilina-eosina (HE), en la cual se evidencia tejido constituido por fibras de colágeno y células fusiformes de núcleo oval y citoplasma no definido, con marcado pleomorfismo celular, núcleos gigantes y numerosas mitosis, la inmunohistoquímica para CD34 y BCL 2 muestra inmunorreactividad de las células; el índice de proliferación ( $\left.\mathrm{K}_{\mathrm{i}}-67\right)$ fue del 60\%. 


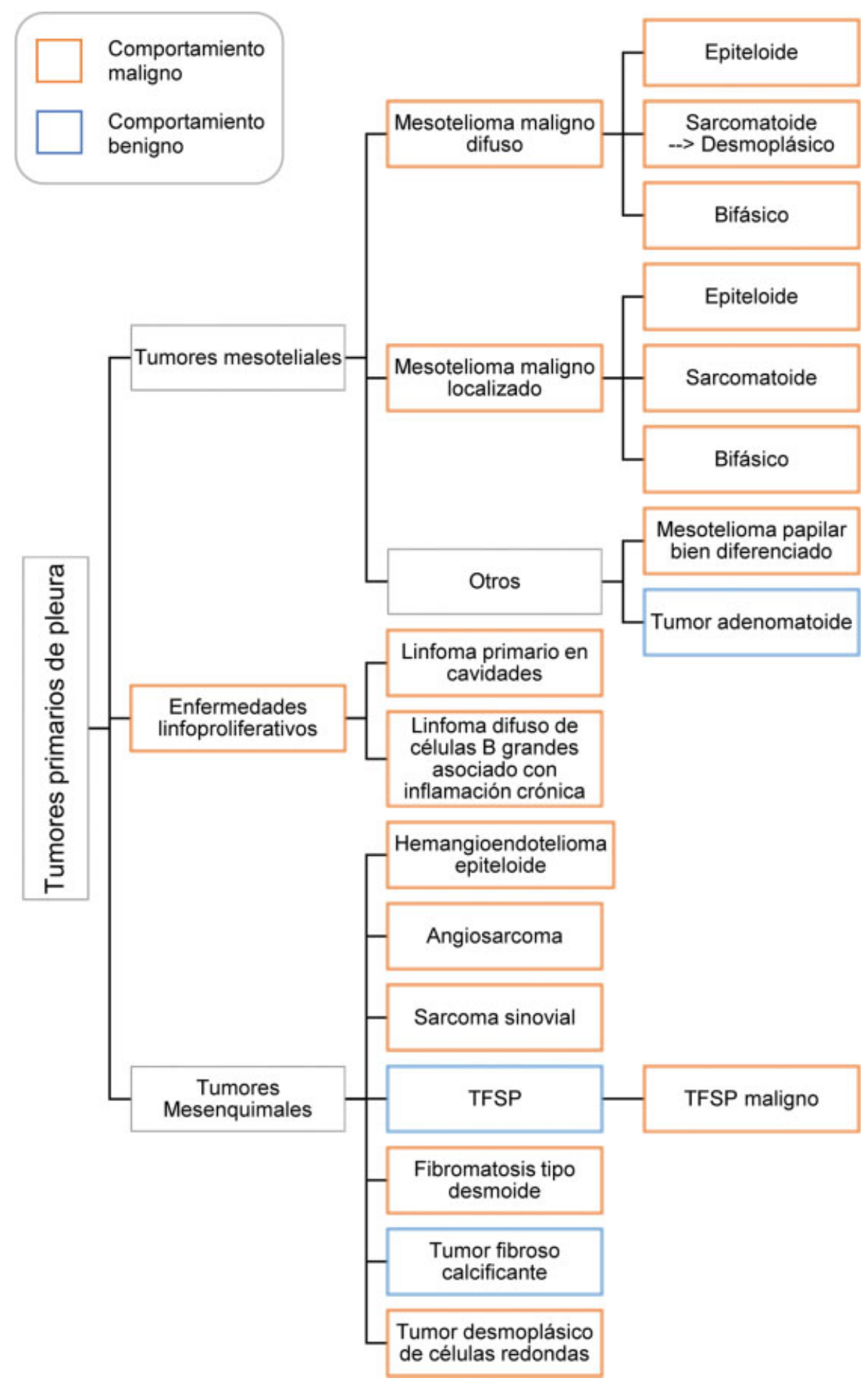

Gráfico 1 Clasificación de los tumores primarios de pleura.

un nódulo o masa de predominio basal y características extrapulmonares, excepto en los casos de localización intracisural, que pueden interpretarse erróneamente como una lesión intrapulmonar.

La TC de pacientes con TFSP evidencia un nódulo o masa sólida, de contornos bien definidos y densidad variable (homogénea en tumores pequeños y heterogénea en lesiones de mayor tamaño), que suele localizarse en las bases de los hemitórax. Generalmente, la lesión no se acompaña de adenomegalias dado el comportamiento benigno en la mayoría de los casos. ${ }^{2,7,15}$

El $42 \%$ de pacientes cursa con uno o más pedículos ricamente vascularizados, que se pueden visualizar de forma más precisa en angiografía. ${ }^{5,16}$ En nuestra serie, durante el procedimiento quirúrgico se demostró que todos los tumores presentaban pedículos (25\% pedículo único y $75 \%$ múltiples). Sin embargo, solo en dos casos se podían visualizar los pedículos en la TC prequirúrgica. 
El 13\% de los TFSP pueden presentar componente quístico de tamaño variable, especialmente en la base del tumor. ${ }^{16}$

La ultrasonografía permite definir la lesión, pero su caracterización y relación con estructuras adyacentes debe realizarse con imágenes axiales. ${ }^{1,2}$

En la serie de 42 casos de Franzen y col., ${ }^{7}$ se describieron como hallazgos frecuentes masa extrapulmonar $(88,1 \%)$, en contacto con la pleura $(88,1 \%)$ y predominio basal $(52,4 \%)$. Otros hallazgos menos frecuentes en el trabajo incluyeron: derrame pleural (11,9\%), desplazamiento de estructuras anatómicas (31\%), invasión de estructuras adyacentes $(4,8 \%)$ y calcificaciones $(11,9 \%){ }^{7}$ A diferencia del estudio anterior, en la serie de Hélage y col., ${ }^{15}$ (56 pacientes) evidenciaron que las calcificaciones (21\%) y el derrame pleural (32\%) son hallazgos frecuentes en los pacientes con TFSP. En este trabajo, el derrame pleural se asoció a malignidad. ${ }^{15}$

En nuestra serie, todos los casos evidenciaron una masa de características extrapulmonares y localización basal. El 75\% de los casos presentó derrame pleural y el 50\% calcificaciones intratumorales.

Los tumores fibrosos se caracterizan por presentar células fusiformes sin patrón y fibras de colágeno. Cuando están localizados en la pleura, ese tipo de tumores suele ser inmunorreactivos a CD34 (90-100\%), BCL-2 (94-100\%) y vimentina $(100 \%)$. Es poco probable encontrar un tumor fibroso con negatividad para CD34 y BCL-2. Por otra parte, los TFSP se caracterizan por una marcación negativa con desmina $(0 \%)$, S100 $(0 \%)$, antígeno epitelial de membrana (0\%), actina de músculo liso $(0 \%)$, citoqueratina $(0-3,5 \%)$, CD117 (3,4\%) y calretinina (0-13\%). La citoqueratina y la calretinina son de utilidad para diferenciar TFSP y mesotelioma. El mesotelioma suele presentar inmunorreactividad para esos marcadores. ${ }^{17}$

Los marcadores inmunohistoquímicos son una herramienta útil para definir pronóstico y naturaleza del tumor fibroso. La marcación positiva para citoqueratina y negativa para CD34 es sugestiva de malignidad o desdiferenciación. ${ }^{17}$ Sin embargo, en el caso presentado de TFSP maligno (caso 3) se documentó inmunorreactividad para CD34.

Un incremento en el índice $\mathrm{K}_{\mathrm{i}}-67$ y la expresión de p53 puede corresponder a un tumor desdiferenciado. La presencia de actividad mitótica, celularidad elevada o necrosis son marcadores de malignidad y pueden predecir un comportamiento tumoral agresivo. ${ }^{17}$

Usualmente, el TFSP es benigno y tiene un buen pronóstico. ${ }^{7}$ Sin embargo, se ha documentado que entre el $10-20 \%$ de los tumores son de naturaleza maligna y pueden presentar recaída local, metástasis y aumento de la mortalidad. ${ }^{2,7,15}$ Se han reportado diversos factores predictores de malignidad: clínicos, radiológicos y patológicos (- Tabla 2).

Los TFSP gigantes suelen ser heterogéneos, el estudio histológico prequirúrgico puede arrojar falsos negativos para malignidad. Al igual que lo reportado en la literatura, en nuestra serie de casos encontramos un paciente (caso 3) a quien se le realizó biopsia, la cual no mostró signos de malignidad. Sin embargo, al resecar la lesión en su totalidad se evidenció una zona de alta celularidad que
Tabla 2 Factores predictores de malignidad $2,3,7,15,16$

\begin{tabular}{|l|}
\hline Clínicos \\
- Sintomatología significativa \\
\hline Radiológicos \\
- Tamaño de la masa $>10 \mathrm{~cm}$ \\
- Derrame pleural \\
- Localización atípica \\
- Heterogeneidad tumoral \\
- Áreas de atenuación hídrica intratumoral \\
- Hipervascularización \\
- Vasos sanguíneos apreciables en TC \\
\hline Macroscópicos \\
- Tamaño de la masa $>10 \mathrm{~cm}$ \\
- Necrosis \\
\hline Histológicos \\
- Necrosis \\
- Alta tasa de mitosis \\
o $>4$ mitosis por 10 campos de alta potencia \\
- Pleomorfismo nuclear \\
- Ki-67 $>12 \%$ \\
- Bordes quirúrgicos positivos \\
\hline
\end{tabular}

correspondía a un foco de tejido maligno. Por lo anterior, para confirmar la naturaleza de la lesión es necesario analizar el tumor en su totalidad. ${ }^{11,18}$

La hipervascularización, definida por presencia de vasos sanguíneos al interior del tumor y aumento en la captación del contraste con respecto a la densidad de los músculos paraespinales, sugiere malignidad. ${ }^{15,16}$ Otro factor pronóstico ampliamente estudiado es el tamaño de la masa. El término "tumor fibroso gigante de pleura," se definió previamente como una lesión superior a $15 \mathrm{~cm}$ de diámetro mayor o que ocupa más del $40 \%$ del hemitórax afectado. No obstante, no hay evidencia suficiente que soporte esa denominación. El TFSP gigante puede presentar características particulares que incluyen: hipercelularidad, necrosis y hemorragia. ${ }^{4,19}$ En nuestra serie, se evidenció necrosis en dos casos, uno de ellos el tumor fibroso de naturaleza maligna y hemorragia en el 50\% de las lesiones.

El tratamiento de elección para el TFSP es la resección quirúrgica (toracotomía/toracoscopia). Un borde de sección de tejido sano $(1-2 \mathrm{~cm})$ disminuye la probabilidad de recurrencia tumoral. ${ }^{2}$

La naturaleza hipervascular del tumor se asocia a complicaciones quirúrgicas como hemorragia. ${ }^{2}$ En tumores de gran tamaño se plantea la posibilidad de embolización prequirúrgica con el objetivo de disminuir el sangrado intraoperatorio y generar edema perilesional inducido por isquemia, el cual facilita la disección tumoral de las estructuras adyacentes. Algunos autores sugieren realizar angiotomografía previa a la embolización percutánea para garantizar la seguridad del procedimiento percutáneo. El tiempo entre la embolización y el procedimiento quirúrgico no debe superar 24 horas. ${ }^{19-21}$

Los tratamientos adyuvantes (quimioterapia radioterapia) postoperatorios no han mostrado ningún beneficio en los casos de TFSP malignos. ${ }^{3}$ 


\section{Conclusión}

Tradicionalmente, se considera que los TFSP son benignos. Sin embargo, es importante considerar la posibilidad de malignidad en pacientes con TFSP de gran tamaño, con manifestaciones clínicas, heterogeneidad de la lesión en los estudios de imágenes e índice de proliferación elevado en el estudio histopatológico. La exclusión definitiva de malignidad en pacientes con TFSP se establece únicamente con el análisis completo de la pieza quirúrgica y el seguimiento de los pacientes. 2,7,15

El tratamiento de elección es quirúrgico y algunos pacientes se pueden beneficiar de embolización prequirúrgica. ${ }^{2,20}$ El seguimiento de los casos de TFSP gigantes debe ser más riguroso, ya que cursan con peor pronóstico y una mayor probabilidad de transformación maligna, en comparación con pacientes que presentan tumores de menor tamaño.

\section{Responsabilidades Éticas}

Protección de personas y animales. Los autores declaran que para esta investigación no se han realizado experimentos en seres humanos ni en animales.

Confidencialidad de los datos. Los autores declaran que han seguido los protocolos de su centro de trabajo sobre la publicación de datos de pacientes.

Derecho a la privacidad y consentimiento informado. Los autores declaran que en este artículo no aparecen datos de pacientes.

Conflictos de Intereses

Los autores declaran no tener conflictos de intereses.

\section{Referencias}

1 Ge W, Yu DC, Chen G, Ding YT. Clinical analysis of 47 cases of solitary fibrous tumor. Oncol Lett. 2016;12(04):2475-2480

2 Abu Arab W. Solitary fibrous tumours of the pleura. Eur J Cardiothorac Surg. 2012;41(03):587-597

3 van Houdt WJ, Westerveld CMA, Vrijenhoek JEP, van Gorp J, van Coevorden F, Verhoef Cet al.. Prognosis of solitary fibrous tumors: a multicenter study. Ann Surg Oncol. 2013;20(13):4090-4095

4 Abe M, Nomori H, Fukazawa M, Sugimura H, Narita M, Takeshi A. Giant solitary fibrous tumor of the pleura causing respiratory insufficiency: report of 3 cases. Ann Thorac Cardiovasc Surg. 2014;20:441-444

5 Rosado-de-Christenson ML, Abbott GF, McAdams HP, Franks TJ, Galvin JR. From the archives of the AFIP: Localized fibrous tumor of the pleura. Radiographics. 2003;23(03):759-783

6 Galateau-Salle F, Churg A, Roggli V, Travis WDWorld Health Organization Committee for Tumors of the Pleura. The 2015 world health organization classification of tumors of the pleura: Advances since the 2004 Classification. J Thorac Oncol. 2016;11(02):142-154

7 Franzen D, Diebold M, Soltermann A, Schneiter D, Kestenholz P, Stahel R, et al. Determinants of outcome of solitary fibrous tumors of the pleura: an observational cohort study. BMC Pulm Med. 2014; $14: 138$

8 Ershadi R, Rahim M, Abbasi M, Erfanian R. Giant solitary fibrous tumor of the pleura. J Surg Case Rep. 2018;2018(11): rjy270

9 Guinee DG, Allen TC. Primary pleural neoplasia: entities other than diffuse malignant mesothelioma. Arch Pathol Lab Med. 2008;132(07):1149-1170

10 Robinson DR, Wu Y-M, Kalyana-Sundaram S, Cao X, Lonigro RJ, Sung YS, et al. Identification of recurrent NAB2-STAT6 gene fusions in solitary fibrous tumor by integrative sequencing. Nat Genet. 2013;45(02):180-185

11 Guo J, Chu X, Sun YE, Zhang L, Zhou N. Giant solitary fibrous tumor of the pleura: an analysis of five patients. World J Surg. 2010;34 (11):2553-2557

12 Meng W, Zhu HH, Li H, Wang G, Wei D, Feng X. Solitary fibrous tumors of the pleura with Doege-Potter syndrome: a case report and three-decade review of the literature. BMC Res Notes. 2014; 7:515

13 Moat NE, Teale JD, Lea RE, Matthews AW. Spontaneous hypoglycaemia and pleural fibroma: role of insulin like growth factors. Thorax. 1991;46(12):932-933

14 de Perrot M, Fischer S, Bründler MA, Sekine Y, Keshavjee S. Solitary fibrous tumors of the pleura. Ann Thorac Surg. 2002;74 (01):285-293

15 Hélage S, Revel MP, Chabi ML, Audureau É, Ferretti G, Laurent F, et al. Solitary fibrous tumor of the pleura: Can computed tomography features help predict malignancy? A series of 56 patients with histopathological correlates. Diagn Interv Imaging. 2016;97(03):347-353

16 England DM, Hochholzer L, McCarthy MJ. Localized benign and malignant fibrous tumors of the pleura. A clinicopathologic review of 223 cases. Am J Surg Pathol. 1989;13(08):640-658

17 Geramizadeh B, Marzban M, Churg A. Role of immunohistochemistry in the diagnosis of solitary fibrous Tumor, a review. Iran J Pathol. 2016;11(03):195-203

18 Gupta A, Souza CA, Sekhon HS, Gomes MM, Hare SS, Agarwal PP, et al. Solitary fibrous tumour of pleura: CT differentiation of benign and malignant types. Clin Radiol. 2017;72(09):796. e9-796.e17

19 Perrotta F, Cerqua FS, Cammarata A, Izzo A, Bergaminelli C, Curcio $\mathrm{C}$, et al. Integrated therapeutic approach to giant solitary fibrous tumor of the pleura: report of a case and review of the literature. Open Med (Wars). 2016;11(01):220-225

20 Khairy M, Othman MH, Ali EM, Eldin EN. Preoperative embolization in surgical management of massive thoracic tumors. Asian Cardiovasc Thorac Ann. 2012;20(06):689-693

21 Weiss B, Horton DA. Preoperative embolization of a massive solitary fibrous tumor of the pleura. Ann Thorac Surg. 2002;73 (03):983-985 